\title{
USO DEL SUELO Y ESTRUCTURA DE LA VEGETACIÓN EN PAISAJES FRAGMENTADOS EN LA AMAZONIA, COLOMBIA
}

\author{
Land use and vegetation structure in forest remnants \\ of fragmented landscapes in Amazonia, Colombia
}

\author{
María Constanza Meza Elizalde y Dolors Armenteras Pascual²
}

Meza-Elizalde, M. C. y Armenteras, D. (2018). Uso del suelo y estructura de la vegetación en paisajes fragmentados en la amazonia, Colombia. Colombia Forestal, 21(2), 205-223

Recepción: 24 de julio de 2017

\section{Resumen}

El seguimiento al uso del suelo y el conocimiento del estado de la vegetación en remanentes de bosques de paisajes representan una metodología fundamental para la planificación del territorio ante la acelerada fragmentación. Por ello, se realizó un análisis multitemporal de la composición y configuración del paisaje (1990-2016) y se establecieron transectos de muestreo de la vegetación. Se identificó una pérdida del $56.34 \%$ de áreas de bosque, aumento en el número de parches, reducción de su área y ampliación de la distancia entre relictos, lo que evidencia procesos activos de fragmentación. En los bosques muestreados se registró la dominancia de especies heliófitas generalistas de ecosistemas perturbados y se encontró una reducción en la complejidad estructural asociada a una baja densidad de individuos (DAP $\geq 10 \mathrm{~cm}$ ) en el estrato superior arbóreo, mostrando que la fragmentación del paisaje ha conllevado a la degradación de estos bosques.

Palabras clave: análisis multitemporal, bosque húmedo tropical, deforestación, degradación forestal, fragmentación, Guaviare, métricas del paisaje.
Aprobación: 22 de marzo de 2018

\begin{abstract}
Monitoring land use and knowledge about the vegetation state in forests remnants which are in a rapid fragmentation process are essential to support decision-making on land planning. We undertook a multitemporal analysis of land use change and landscape configuration for the period 1990-2016. Vegetation sampling transects were established in three forest relicts. Our results indicate a loss of $56.34 \%$ of forest area, an increase in the patches number, area reduction and a distance increase between the forest fragments. In the sampled forests, the dominance of generalist heliophyte species from disturbed ecosystems was found. Also, we observed a structural complexity reduction of the forest associated with an individuals low density with a $\mathrm{DBH} \geq 10 \mathrm{~cm}$ in the upper tree layer, showing that landscape fragmentation has led to the forest degradation.
\end{abstract}

Key words: multitemporal analysis, tropical rain forest, deforestation, forest degradation, fragmentation, Guaviare, landscape metrics.

1 Grupo de Investigación en Ecología del Paisaje y Modelación de Ecosistemas (Ecolmod). Universidad Distrital Francisco José de Caldas. Bogotá D.C, Colombia. mcmesae@correo.udistrital.edu.co. Autor para correspondencia.

2 Grupo de Investigación en Ecología del Paisaje y Modelación de Ecosistemas (Ecolmod). Universidad Nacional de Colombia. Bogotá D.C, Colombia.darmenterasp@unal.edu.co 


\section{INTRODUCCIÓN}

El paisaje es el producto de numerosas interacciones entre los factores bióticos, abióticos y sociales (Torres-Gómez, Delgado, Martín y Bustamante, 2009), en el cual el uso y manejo del territorio es el factor más determinante en los procesos de deforestación, fragmentación y degradación de los bosques (Armenteras y Vargas, 2016), principalmente en las zonas de fácil acceso (Vergara y Gayoso, 2004). Es por esto que el uso del suelo realizada por el hombre la hace más simple y con mayor homogeneidad en el paisaje, constituyéndose en el principal determinante de las medidas del paisaje y el indicador más sensible de los sistemas terrestres (Peng, Mi, Qing y Xue, 2016).

Las actividades del hombre han Ilevado al deterioro de los bosques debido a la expansión de la frontera agrícola, la ganadería, la infraestructura y la minería; y otras causas indirectas asociadas a los cambios sociales, políticos y económicos, como lo son el crecimiento demográfico, la tenencia de la tierra y políticas sectoriales (Armenteras y Rodriguez, 2014).

Las causas de deforestación varian según el tipo de bosque. Sin embargo, en la mayoría de países latinoamericanos las principales causas de deforestación son el acceso a los mercados, las actividades agrícolas y forestales (Armenteras, Espelta, Rodriguez y Retana, 2017). En el caso de los bosques amazónicos, la extracción de madera, la expansión de cultivos y las áreas de pastos dedicadas al pastoreo extensivo constituyen los principales motores de deforestación (Martino, 2007). Se ha sugerido que a medida que aumenta la apertura de comercio la deforestación también ha aumentado (Rodriguez y Nunes, 2016).

Se logran diferenciar de forma marcada dos patrones de deforestación en toda la región amazonica: uno geométrico o difuso asociado a la consolidación de las fronteras de colonización y otro en espina de pescado asociado a la construcción de carreteras (Armenteras y Rodriguez, 2014). Por ejemplo, en la Amazonia brasilera y algunos sectores de la ecuatoriana, los principales ejes de deforestación son las carreteras (Rodriguez y Nunes, 2016); en Brasil se presenta una tendencia fuerte de deforestación influenciada por políticas sectoriales asociadas a hidrocarburos, infraestructura para la producción de energía y cultivos intensivos de soja y caña de azúcar (Martino, 2007); mientras que en Ecuador la palma africana para la obtención de aceite es el motor que impulsa la pérdida de bosques amazónicos (Potter, 2011). En países como Perú, Bolivia y Colombia se les suma los cultivos ilícitos como causantes de deforestación (Martino, 2007).

En la Amazonia colombiana el patrón de deforestación y fragmentación está asociado a los ríos dado que estos son el medio principal de colonización y desarrollo (Armenteras, Rudas, Rodríguez, Sua y Romero, 2006), y se presenta una alta tasa de conversión de bosques a pastos (Armenteras et al., 2006; Murcia, Huertas, Rodríguez y Castellanos, 2011; Dávalos, Holmesb, Rodriguez y Armenteras, 2014). Entre los años 2005 y 2010, el primer factor de pérdida de área boscosa y fragmentación de la Amazonia colombiana fue la transformación de bosques a pastos (Dane, 2013), siendo el departamento de Guaviare uno de los principales focos de deforestación en la zona del piedemonte amazonense, registrando pérdidas de bosques en mayor cantidad y de forma más acelerada (Nepstad et al., 2013). El Guaviare tiene una tasa media anual de deforestación de 65013.43 ha.año-1 (Murcia et al., 2011), y de los cuatro municipios que lo constituyen (Calamar, El Retorno, Miraflores y San José del Guaviare) los que presentan mayor tasa de deforestación son San José del Guaviare y El Retorno, con una tasa anual de deforestación de 9364.9 y 6492.65 ha año-1, respectivamente (Murcia et al., 2011).

Un segundo suceso a la tasa de deforestación es el grado relativo de fragmentación de los ecosistemas naturales (Armenteras et al., 2006), que puede verse con la expansión de la frontera agropecuaria en la cual se generan numerosos fragmentos de bosque inmersos en una matriz donde predominan sistemas agrícolas y ganaderos (Chacón, Harvey y 
Delgado, 2008). El aumento en el número de fragmentos, la reducción de su área y el aumento en la distancia entre estos, son una limitante para algunos procesos ecológicos como la dispersión de semillas, la colonización, la migración y la interacción entre especies (Matteucci, 2004). Todos estos sucesos conllevan a cambios de amplio alcance en la recomposición de comunidades forestales, generando tensiones entre las especies de plantas tolerantes a la sombra y favoreciendo la regeneración de árboles pioneros (Laurance et al., 2011), hasta que algunas poblaciones alcancen un umbral por debajo del cual son inviables (Santos y Telleria, 2006). Igualmente, el proceso de fragmentación hace que aumenten la suceptibilidad de los bosques y el riesgo de sufrir impactos (Cordero y Boshier, 2003). Dentro de los impactos más recurrentes de los procesos de fragmentación se encuentra la mayor exposición e influencia de los ambientes periféricos que se evidencia en una interfase entre e1 bosque y su matriz circundante, conocida como borde (Gurrutxaga y Lozano 2008). El borde generado facilita el sobrepastoreo en áreas de bosque (Ormazábal, Ávila, Mena, Morales y Bustos, 2013), la incidencia de incendios (Armenteras, Gonzales y Retana, 2013) y la colonización de plantas invasoras (Bustamante y Grez, 1995; Fuentes-Ramírez, Pauchard, Marticorena y Sánchez, 2010), en definitiva a la degradación de las funciones ecológicas de los ecosistemas (Jiang, Cheng, Li, Zhao y Huang, ,2014).

La afectación de los ecosistemas se evidencia en la reducción de la calidad de la vegetación que implica cambios en su composición y estructura, como la dominancia de pocas especies, la abertura del dosel y regeneración escasa de plantas nativas tolerantes a la sombra (Acharya, Dangi y Acharya, 2011; Thompson, 2011), colocando en riesgo la conservación de la biodiversidad al interior de los relictos (Chacón et al., 2008) e indicando pérdida de resilencia con una reducción en la producción de bienes y servicios (Acharya et al., 2011).

Es por ello que, considerando la exposición del paisaje a procesos continuos de deforestación por cambios en el uso del suelo, se hace necesario detectar y hacer seguimiento de estos cambios, lo cual puede realizarse a partir del análisis multitemporal de la cobertura del suelo, así como de métricas del patrón espacial (Sun y Zhou, 2016). El análisis de los cambios de cobertura permite detectar y estimar la extensión de los cambios (Canzio, 2006), mientras que la cuantificación de la configuración espacial a través de métricas permite describir la estructura y dinámica de cambio de los elementos estructurales del paisaje (Matteucci, 2004).

Sin embargo, el conocimiento de los cambios de cobertura y estructura del paisaje por sí solos no bastan para confirmar las asociaciones que se dan entre patrones y procesos (Matteucci, 2004). Por lo cual, se hace necesario emplear metodologías alternativas que permitan analizar las relaciones sociales entre la dinámica de transformación del paisaje, los procesos subyacentes que conducen al cambio (Vieira y Castillo, 2010) e investigaciones de procesos ecológicos (Aguilera y Botequilla- Leitão, 2012).

El análisis integrado de los cambios de cobertura, configuración espacial y factores sociales que inciden en ellos son poco frecuentes (Torres-Gómez et al., 2009), por ello, este estudio buscó integrar metodologías a diferentes escalas espaciales y temporales que contribuyan al desarrollo de alternativas de manejo para la conservación planificada de los ecosistemas del departamento del Guaviare. Para lo cual, el objetivo general de este estudio fue identificar si se han presentado procedimientos de fragmentación del paisaje y si estos han conllevado a procesos de degradación que se reflejen en la composición florística y estructural de bosques de galería a escala local.

Para dar cumplimiento al objetivo general de este estudio, los objetivos específicos fueron: realizar un análisis de coberturas del suelo y determinar las principales métricas del paisaje; analizar las relaciones sociales que han influenciado la dinámica de transformación del paisaje; y caracterizar la composición y estructura de relictos boscosos presentes en el área de estudio. 


\section{MATERIALES Y MÉTODOS}

\section{Área de estudio}

El área de estudio (figura 1) se encuentra en el municipio El Retorno del departamento del Guaviare (Colombia), ubicado en la cuenca alta del río Inírida por debajo de los 500 m de altitud, se encuentra en la zona de vida de bosque húmedo tropical (Bh-T), presenta temperaturas mayores a los $24^{\circ} \mathrm{C}$ y precipitaciones que oscilan entre los 2000 y 4000 $\mathrm{mm}$ anuales (Igac, 2008). Hace parte de la reserva forestal de la Amazonia (Ley 2 de 1959), el área de protección regional Ariari-Guayabero y la Zona de Reserva Campesina del Guaviare (ZRCG).

En el ámbito socioeconómico, el área de estudio se ha caracterizado por relaciones económicas extractivas, desiguales y reforzadas por coaliciones violentas acompañadas de desplazamiento y olas de colonización (García, 1995). Su estructura económica se basa en las bonanzas y no en una base económica sustentable, lo cual ha conllevado a que no se consolide aún la frontera agropecuaria y se presente procesos de expansión (Sinchi, 1999). El Retorno ha presentado procesos de colonización campesina que intercala pequeños cultivos de coca (Reyes, 1998), los cuales se mantienen en la actualidad. Sin embargo, según el Informe de Gestión Municipal 2012-2015, la ganadería es la actividad principal por lo cual esta es considerado la capital ganadera del Guaviare. Igualmente, se destacan otras actividades como la producción de caucho, contando con el 50\% de cultivos con más de 1715 ha instaladas en el departamento (Confederación Cauchera Colombiana, 2016).

\section{Entrevistas a actores clave}

Se realizaron siete entrevistas cualitativas semiestructuradas, entre marzo y julio de 2016 a diferentes actores clave de la zona (productores de coca, agricultores familiares, ganaderos, productores de caucho y funcionarios públicos). Los actores clave fueron referenciados por habitantes de la zona y posteriormente fueron contactados de forma directa. Se emplearon dos criterios para la selección de los entrevistados: ser habitantes del municipio con antigüedad mayor a 30 años y desarrollar una actividad productiva en el municipio. Para la orientación de las preguntas se consideraron tres dimensiones: actividades económico-productivas, organización comunitaria y conflictos sociales. Por último, con el fin de contextualizar e interpretar las afirmaciones de los entrevistados, se comparó la información proporcionada con otra obtenida a través de observaciones no partícipes. Con base en lo recolectado, se construyó una línea del tiempo cuyo fin es identificar las dinámicas de cambio en el uso del suelo que podrían estar relacionadas con la ganancia o pérdida de cobertura boscosa.

\section{Análisis multitemporal de imágenes}

Se seleccionaron tres relictos boscosos que no han presentado extracción de productos forestales en los últimos 30 años, alrededor de los cuales se estableció un área buffer de 10 km; suficiente considerando que el área mínima adecuada para observar la disposición de las diferentes clases de uso del suelo, así como obtener diferentes métricas del paisaje (Costafreda, 2009; Arancibia-Arce et al., 2013; Fernández y Silva, 2015). Sobre esta se hizo un análisis multitemporal de las coberturas de la tierra para el periodo comprendido entre los años 1990 y 2016, empleando imágenes Landsat 4 para los años 1990, 1991, 1997 y 2000, imágenes Landsat $7(E T M+)$ para el año 2002 e imágenes Landsat 8 para los años 2013 y 2016; todas estas disponibles en el servidor libre Earth Resources Observation and Science Center (Eros) del Servicio Geológico de los Estados Unidos (USGS). El procesamiento de las imágenes se realizó con el software Impact Toolbox versión 1.3.7 beta (Simonetti, Marelli y Eva, 2015), identificando cinco clases de cobertura (tabla 1) con base en la Leyenda Nacional de Coberturas de la Tierra Corine Land Cover nivel 2 adaptada para Colombia (Ideam, 2010). 

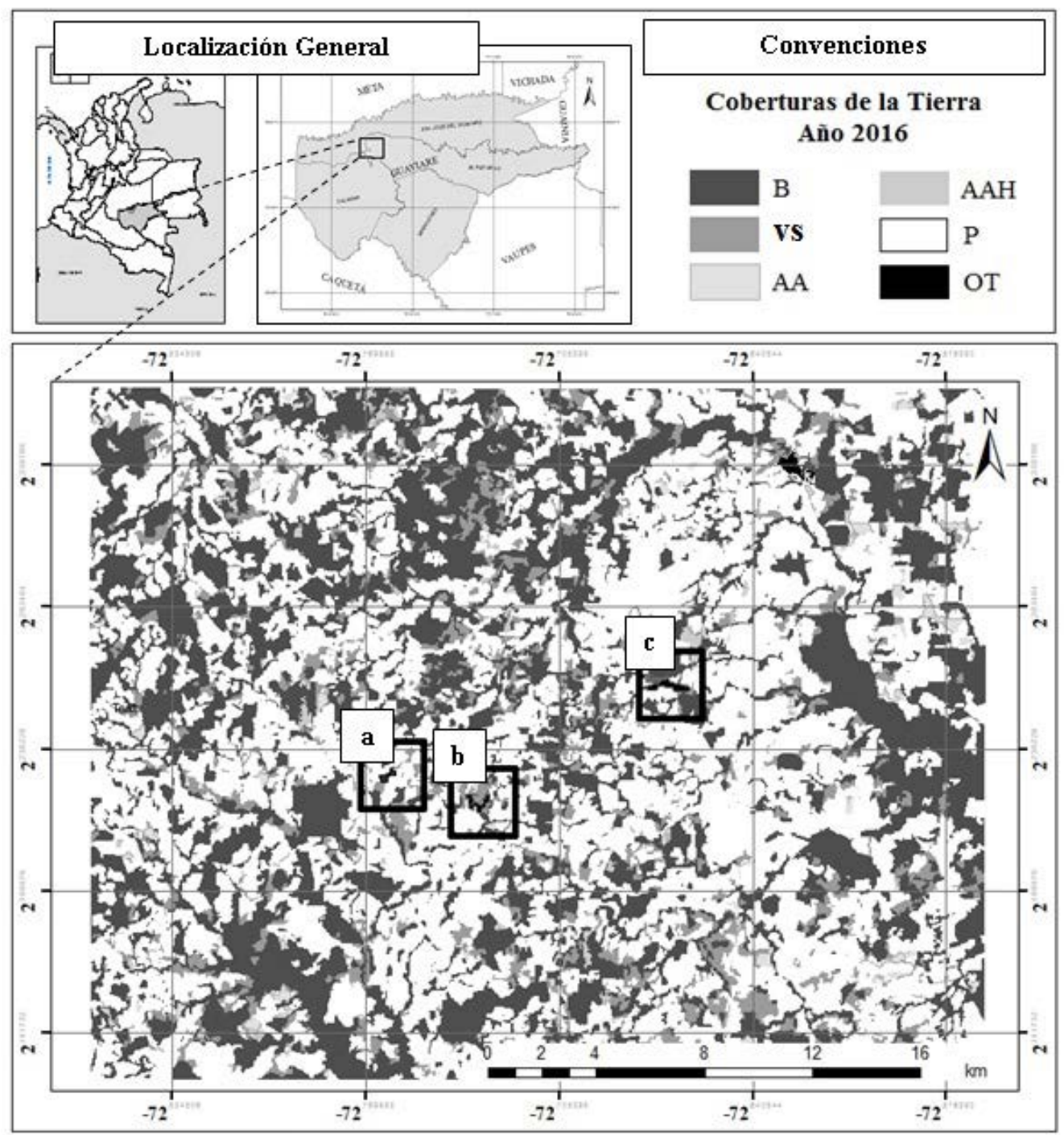

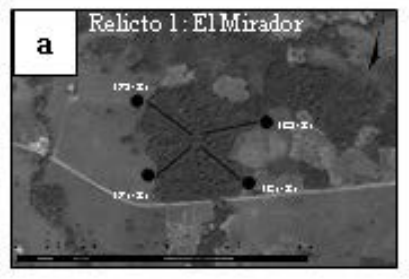

Localización Transectos de Muestreo

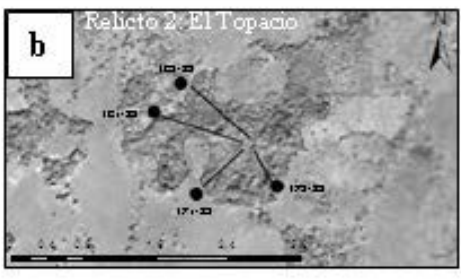

Localización Transectos de Muestreo

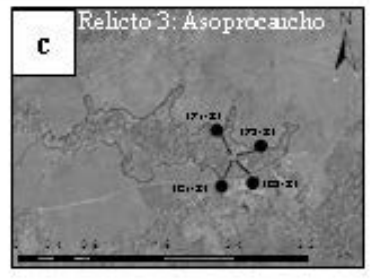

Localización Transectos de Muestreo

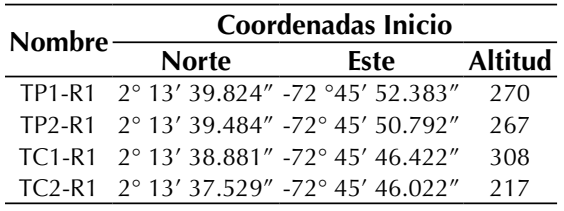

\begin{tabular}{cccc}
\hline \multirow{2}{*}{ Nombre } & \multicolumn{3}{c}{ Coordenadas Inicio } \\
\cline { 2 - 4 } & Norte & Este & Altitud \\
\hline TP1-R2 & $2^{\circ} 13^{\prime} 1.1009^{\prime \prime}-72^{\circ} 43^{\prime} 52.637^{\prime \prime}$ & 281 \\
TP2-R2 & $2^{\circ} 13^{\prime} 0.2741^{\prime \prime}-72^{\circ} 44^{\prime} 1.2313^{\prime \prime}$ & 398 \\
TC1-R2 & $2^{\circ} 13^{\prime} 5.8790^{\prime \prime}-72^{\circ} 44^{\prime} 2.0326^{\prime \prime}$ & 381 \\
TC2-R2 & $2^{\circ} 13^{\prime} 4.0542^{\prime \prime}-72^{\circ} 44^{\prime} 2.9087^{\prime \prime}$ & 384 \\
\hline
\end{tabular}

\begin{tabular}{cccc}
\hline \multirow{2}{*}{ Nombre } & \multicolumn{3}{c}{ Coordenadas Inicio } \\
\cline { 2 - 4 } & Norte & Este & Altitud \\
\hline TP1-R3 & $2^{\circ} 15^{\prime} 26.057^{\prime \prime}-72^{\circ} 39^{\prime} 47.606^{\prime \prime}$ & 215 \\
TP2-R3 & $2^{\circ} 15^{\prime} 24.339^{\prime \prime}-72^{\circ} 39^{\prime} 44.819^{\prime \prime}$ & 220 \\
TC1-R3 & $2^{\circ} 15^{\prime} 20.020^{\prime \prime}-72^{\circ} 39^{\prime} 46.823^{\prime \prime}$ & 204 \\
TC2-R3 & $2^{\circ} 15^{\prime} 20.612^{\prime \prime}-72^{\circ} 39^{\prime} 44.012^{\prime \prime}$ & 224 \\
\hline
\end{tabular}

AA: áreas abiertas, sin o con poca vegetación. AAH: áreas agrícolas heterogéneas. AVHA: áreas con vegetación herbácea y arbustiva. B: bosques. OT: otras tierras. SD: sin datos.

Figura 1. Área de estudio. 
Tabla 1. Clases de cobertura empleadas en el estudio

\section{Clases de cobertura}

Zonas urbanizadas u otras tierras (OT): hace referencia a los territorios cubiertos por infraestructura urbana y todos aquellos espacios verdes y redes de comunicación asociados con ellas.

Pastos (P): comprende tierras ocupadas por pastos con un porcentaje de cubrimiento mayor al $70 \%$. Su presencia se debe a la acción antrópica, por lo cual las prácticas de manejo empleadas impiden el establecimiento de otras coberturas. Son lugares con pastoreo permanente por un periodo de dos o más años. La composición florística dominante son hierbas principalmente de la familia Poaceae.

Áreas agrícolas heterogéneas (AAH): hace referencia a unidades de área que reúnen dos o más clases de coberturas agrícolas o naturales, pero dado el tamaño de estas no pueden ser representadas de forma individual. En estos mosaicos las áreas de pastos y cultivos son dominantes, ocupando entre el $30 \%$ y $70 \%$ de la unidad; en menor medida se pueden encontrar espacios naturales asociados a bosques u otras coberturas naturales.

Vegetación secundaria o en transición (VS): son aquellas áreas cubiertas por vegetación principalmente arbustiva y herbácea con dosel irregular y presencia ocasional de árboles y enredaderas. Corresponden a unidades en estadios iniciales de sucesión vegetal después de presentarse un proceso de deforestación de los bosques o aforestación de los pastizales o áreas agrícolas abandonadas.

Bosques (B): comprende áreas naturales o seminaturales constituidas por elementos arbóreos que alcanzan una altura de dosel superior a los $5 \mathrm{~m}$. La cobertura arbórea ocupa más del $70 \%$ de la unidad.

Áreas abiertas, sin o con poca vegetación (AA): comprende territorios en los cuales la cobertura vegetal no existe o es escasa. Conformado comúnmente por suelos desnudos y quemados.

Fuente: Ideam (2010).

Con el fin de determinar la confiabilidad de las clasificaciones temáticas, se siguió el procedimiento propuesto por Stehman y Czaplewski (1998). Se tomaron como unidad de muestreo puntos de control de diferentes fuentes, la primera referida a 640 puntos capturados con GPS en campo durante los años 2015 y 2016. Como segunda fuente, se estratificó la ventana de estudio en las clase temáticas de áreas de bosque y no bosque, sobre cada una de las cuales se generaron puntos aleatorios simples con el software QGIS v. 2.12.2 (Quantum GIS Development Team, 2016), más de 50 por clase temática, dado que es el mínimo recomendado para evaluar la precisión (Chuvieco, 2000); y se tomó como único criterio la distancia de separación entre puntos, mayor a 400 m. Los puntos creados se validaron con ortofotos de alta resolución y cartografía temática regional a una escala de 1:100 000. Para el análisis de precisión de los años 1990, 2000 y 2013, los puntos fueron comparados con los mapas de cobertura de bosque-no bosque de los mismos años del Instituto de Hidrología, Meteorología y Estudios Ambientales de Colombia (Ideam). El año 2002 se validó con el mapa de cobertura de la tierra de la Amazonia del mismo año del Instituto Amazónico de Investigaciones Científicas (Sinchi); los años 1991 y 1997 se validaron con puntos en áreas boscosas no cambiantes entre los años 1990-2000 apoyados en ortofotos de alta resolución; y, por último, el año 2016 se validó con los puntos control tomados en campo. La exactitud temática de los mapas de cobertura generados se determinó a través de una matriz de confusión empleando el coeficiente de Kappa (Chuvieco, 2000). Para obtener la información más precisa posible se tomó como mínimo permitido un porcentaje de $60 \%$ de coincidencia con respecto a la información de referencia, Ilegando a fiabilidades altas (Landis y Koch, 1977).

\section{Métricas de paisaje}

Con base en la información referida se realizaron los mapas de coberturas para los años 1989 y 2016, los cálculos de métricas de área, forma y agregación a nivel de clase (tabla 2), empleando el software Fragstats v. 4.2.1. (McGarigal, Cushman y Ene, 2012). 
Tabla 2. Métricas de paisaje empleadas a los niveles de clase

\begin{tabular}{|c|c|}
\hline Tipo & Descripción \\
\hline \multirow{4}{*}{ Área } & $\begin{array}{l}\text { Área (CA): suma de todas las áreas de todos los parches de un tipo de cobertura }\left(\mathrm{m}^{2}\right) \text {, dividido por } 10000 \text { para } \\
\text { convertirlo a hectárea. }\end{array}$ \\
\hline & Número de parches (NP): número de parches de cada clase de cobertura. \\
\hline & $\begin{array}{l}\text { Densidad de parches (DP): muestra el número de parches por área (100 ha), permite determinar comparacio- } \\
\text { nes de mosaicos en diferentes tamaños y niveles de fragmentación. }\end{array}$ \\
\hline & $\begin{array}{l}\text { Índice de parche más grande (LPI): indica el porcentaje de área que ocupa el parche grande de cada clase } \\
\text { sobre toda el área de estudio. Este indicador es útil para realizar comparaciones de la evolución de los parches } \\
\text { mayores en el paisaje para diferentes años. Toma valores de } 0 \text { a } 100(\%) \text {. }\end{array}$ \\
\hline Forma & $\begin{array}{l}\text { Proporción perímetro/área (Para): razón entre la longitud del parche }(\mathrm{m}) \text { y el área }\left(\mathrm{m}^{2}\right) \text {, es una medida de } \\
\text { complejidad de forma. }\end{array}$ \\
\hline \multirow{2}{*}{ Agregación } & $\begin{array}{l}\text { Distancia euclidiana al vecino más cercano (ENN): distancia al parche más cercano del mismo tipo (m), basa- } \\
\text { do en la distancia de borde a borde más corta. Es útil para cuantificar aislamiento entre parches. Valores más } \\
\text { altos indican mayor aislamiento entre parches de la misma clase de cobertura. }\end{array}$ \\
\hline & $\begin{array}{l}\text { Índice de agregación }(\mathbf{A I}) \text { : número de adyacencia de cada clase de cobertura dividido entre el máximo núme- } \\
\text { ro de adyacencias posibles. Toma un valor de } 0 \text { para una mínima agregación y } 100 \text { para la máxima agrega- } \\
\text { ción. }\end{array}$ \\
\hline
\end{tabular}

Fuente: McGarigal y Marks (1995) y Matteucci (2004).

\section{Composición y estructura de la vegetación}

En cada uno de los relictos de estudio (tres), se establecieron cuatro transectos variables de Foster (Cámara y Díaz del Olmo, 2013), con un ancho fijo de $10 \mathrm{~m}$ y una longitud de $100 \mathrm{~m}$, en estos se hizo la medición de fustales (DAP $\geq 10 \mathrm{~cm} ; \mathrm{HT}>1.5$ $\mathrm{m})$. Cada transecto se subdividió en transectos de igual longitud al principal, pero con un ancho de $4 \mathrm{~m}$, para la medición de latizales $(\mathrm{DAP}<10 \mathrm{~cm}$; HT > $1.5 \mathrm{~m}$ ). Para la medición de la regeneración natural, a lo largo de los transectos se establecieron parcelas cuadradas de $4 \mathrm{~m}^{2}$, separadas entre sí por $10 \mathrm{~m}$, en las cuales se midieron brinzales (DAP < $10 \mathrm{~cm} ; \mathrm{HT}>0.3 \mathrm{~m}), y$ estas a su vez se subdividieron en parcelas de $1 \mathrm{~m}^{2}$ para hacer el conteo de renuevos (DAP $<10 \mathrm{~cm} ; \mathrm{HT}<0.3 \mathrm{~m}$ ). Se determinó el índice de valor de importancia ampliado (Ivia), combinando la estructura horizontal y vertical de todas las categorías de tamaño para determinar las especies con mayor peso ecológico en el bosque. Además, se realizó la distribución por clase diamétrica y altimétrica para todos los individuos con DAP $\geq 10 \mathrm{~cm}$ (fustales); y todos los individuos de las categorías de tamaño de fustales y latizales se estratificaron en tres categorías de acuerdo a su altura total para determinar la altura dominante del bosque (Gadow, Real y Álvarez, 2001).

Se colectaron muestras botánicas de todos los individuos muestreados. Para la determinación del material vegetal se recurrió a la consulta de claves taxonómicas, literatura e información existente en colecciones botánicas, así como a la comparación directa con los ejemplares de la colección del Herbario Forestal Gilberto Emilio Mahecha Vega (UDBC) de la Universidad Distrital Francisco José de Caldas.

\section{RESULTADOS}

\section{Análisis multitemporal del cambio de uso de suelo:}

La exactitud temática de los mapas de cobertura generados tiene un porcentaje mayor al $60 \%$ de coincidencia con respecto a la información de referencia, por lo que alcanza una alta fiabilidad con un valor de Kappa que varía entre 0.64 y 0.91 para 
la clasificación de todas las coberturas. Se encontró que la clasificación del año 2016 validada con puntos de referencia tomados en campo obtuvo la mayor precisión, con un coeficiente de Kappa de 0.908. Los valores obtenidos y la fuerza de concordancia se presentan en la tabla 3.

En el año 1990 los bosques predominaban la cobertura ocupando el $67.24 \%$ del área de estudio, mientras que en el año 2016 representaban el $37.88 \%$; los pastos limpios dedicados a la ganadería extensiva tenían la cobertura dominante del el $50.27 \%$ del área de estudio (figura 2). Con base en la información suministrada por los entrevistados, se identificó a la ganadería extensiva y los cultivos ilícitos como las actividades que han impulsado en mayor medida la pérdida de bosques en el área de estudio y su dinámica se encuentra directamente relacionado con el conflicto armado (figura 3). Al contrastar esta información con los resultados del análisis multitemporal, se encontró que durante los años 1991 y 1997, en los cuales se implementaron en el municipio otras estrategias productivas como los cultivos de caucho, se registraron menos hectáreas de tierra dedicadas a los pastos. Igualmente, se identificaron dos periodos en los cuales la pérdida de área boscosa fue mayor, el primero comprendido entre 1997 y 2000, años en los que se presentaron gran cantidad de cambios en la dinámica del cultivo de coca; y un segundo periodo desde 2002 hasta 2013, coincidiendo con la época de mayor desplazamiento de la población a causa del conflicto armado y la compra de grandes extensiones de tierra para la ganadería extensiva.

Los entrevistados también señalaron el rol de las plantaciones de caucho como actividad productiva para la conservación de las áreas de bosque e indicaron que esta actividad obtuvo su mayor auge a inicios de los años 90 través del Plan de Desarrollo Alternativo (Plante); (1993-1996), como alternativa de sustitución de cultivos ilícitos que venían proliferando desde la década del 70 y que se vio frenado por el desplazamiento de la población a causa del conflicto armado. Los entrevistados relacionaron que la apuesta productiva de la región en la actualidad está orientada al establecimiento de cultivos de caucho y cacao, así como de ganadería sostenible, en aras de promover la conservación de los bosques; iniciativas que se han mantenido desde el año 2008, cuando surgió el Programa de Alianzas Productivas.

En lo concerniente a las métricas de paisaje, se encontró que al comparar los años 1990 con 2016 hay un aumento significativo en el número de parches de bosques que pasó de 62 (1990) a 339 (2016); mientras que la cobertura de pastos disminuyó la cantidad de parches de 552 a 332, respectivamente. Así, son los bosques los que en 1990 tenían los parches con mayor área con un valor medio de 918.89 ha, mientras que en el 2016 el área promedio de los parches de bosque es de 94.68 ha. Los pastos, por su lado, presentan mayor porcentaje de área ocupada dentro del paisaje con un área promedio de parches con 128.29 ha.

Tabla 3. Valores de coeficiente de Kappa para el análisis multitemporal del uso del suelo en El Retorno (Colombia)

\begin{tabular}{ccccc}
\hline Imagen & $\begin{array}{c}\text { Coeficiente } \\
\text { de Kappa }\end{array}$ & $\begin{array}{c}\text { Error } \\
\text { estándar }\end{array}$ & I.C (95\%) & $\begin{array}{c}\text { Fuerza de la } \\
\text { concordancia }\end{array}$ \\
\hline $08 / 01 / 1990$ & 0.640 & 0.045 & $(0.552-0.728)$ & Buena \\
$16 / 03 / 1991$ & 0.693 & 0.057 & $(0.581-0.805)$ & Buena \\
$13 / 12 / 1997$ & 0.647 & 0.060 & $(0.529-0.764)$ & Buena \\
$13 / 02 / 2000$ & 0.747 & 0.052 & $(0.645-0.849)$ & Buena \\
$30 / 09 / 2002$ & 0.661 & 0.052 & $(0.559-0.764)$ & Buena \\
$06 / 10 / 2013$ & 0.649 & 0.068 & $(0.515-0.782)$ & Buena \\
$01 / 02 / 2016$ & 0.908 & 0.090 & $(0.731-1.085)$ & Muy buena \\
\hline
\end{tabular}

I.C: índide de concordancia. 


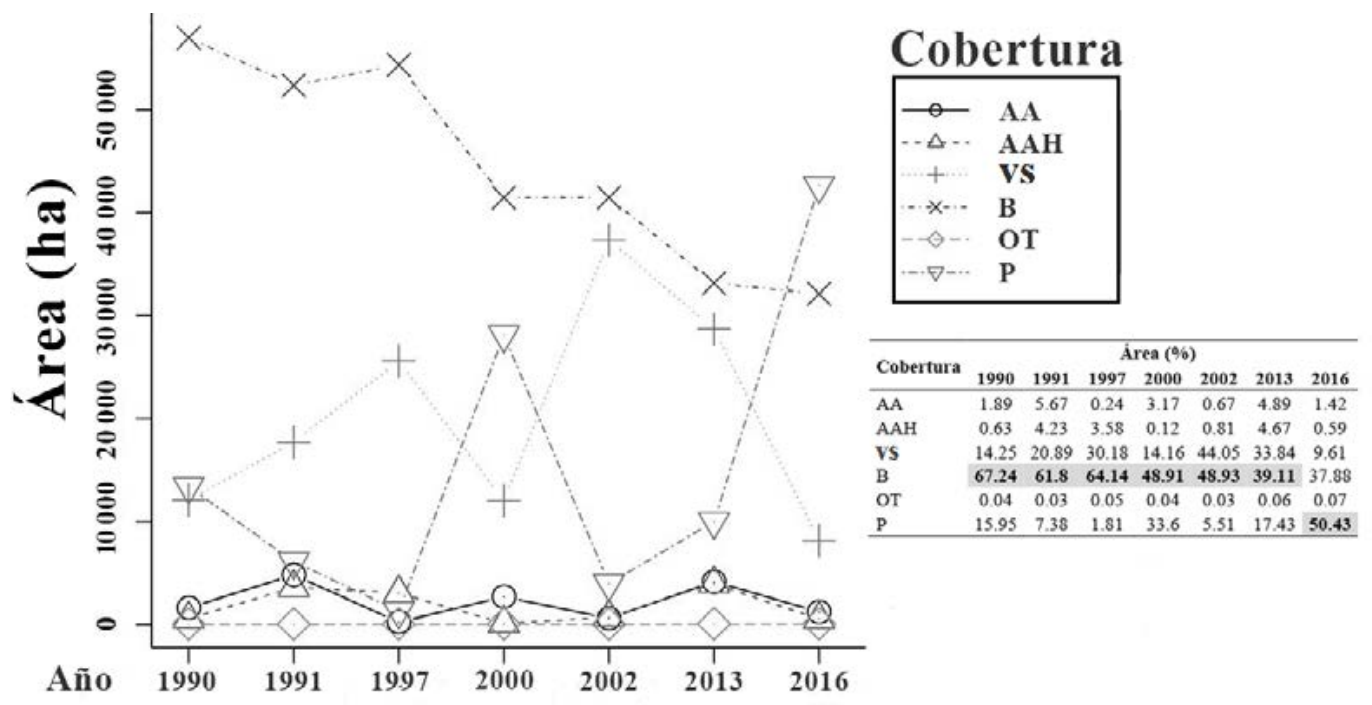

AA: áreas abiertas, sin o con poca vegetación. AAH: áreas agrícolas heterogéneas. AVHA: áreas con vegetación herbácea y arbustiva. B: bosques. OT: otras tierras. SD: sin datos.

Figura 2. Área (ha) por clasesde cobertura en el periodo 1990-2016.

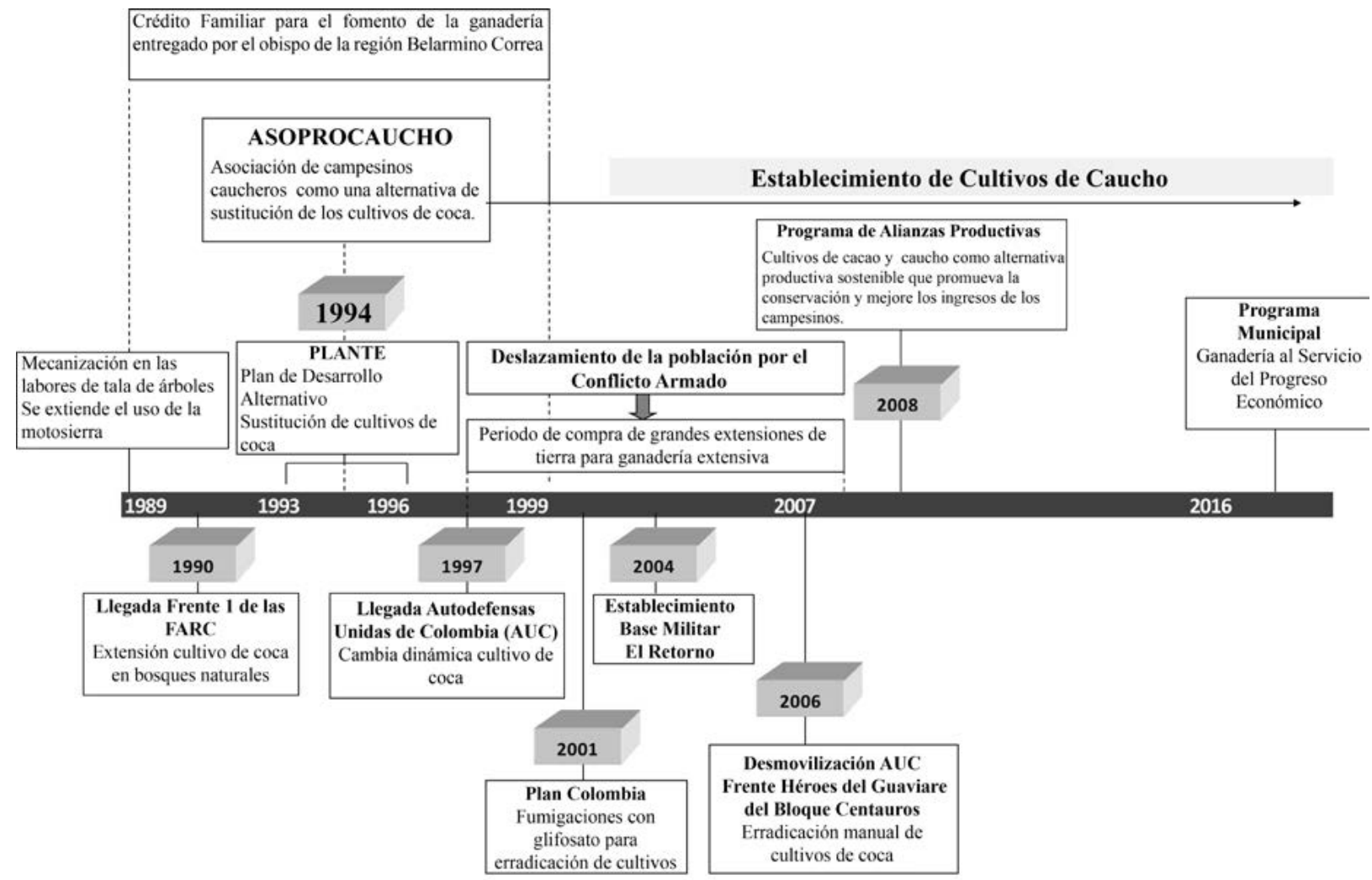

Figura 3. Línea del tiempo para el periodo comprendido entre 1990-2016. Elementos socioeconómicos que han incidido en el cambio de uso del suelo. 
En relación al índice del parche más grande, en 1990 los bosques (64.84\%) eran la cobertura que tenían los parches con mayor área, mientras que en 2016 las coberturas de bosques y pastos son las que tienen mayor porcentaje de área ocupada por su parche más grande dentro del paisaje, con $15.06 \%$ y $11.76 \%$, respectivamente.

En lo que respecta a las mediciones de la forma de los fragmentos, de acuerdo al índice de proporción perímetro/área (Para), se evidencia que, dada la relación entre la longitud del parche $(\mathrm{m})$ y el área $\left(\mathrm{m}^{2}\right)$, de 1990 al 2016 aumentó la relación para los bosques mientras que disminuyó el valor del índice para los pastos.

De acuerdo a las métricas de agregación, y considerando la distancia media de los fragmentos al vecino cercano (ENN), los bosques presentaron un aumento de $7.39 \mathrm{~m}$, lo cual muestra una mayor dispersión de los parches de esta cobertura. Por el contrario, los pastos mostraron una disminución de la distancia entre parches, la cual pasó de 243.26 m en 1990 a 99.38 m en el 2016. El índice de agregación (Al) entre los años 1990 al
2016 presenta una disgregación de fragmentos de bosque mientras que aumentan la agregación de parches de pastos. En la tabla 4 se relacionan las métricas de paisaje a nivel de clase tomadas para los años 1990 y 2016, respectivamente.

\section{Composición florística}

En el relicto 1 (predio El Mirador) se registraron 208 especies forestales pertenecientes a 55 familias y se encontró que las más representativas son: Moraceae con 14 especies, Rubiaceae con 13 especies y Burseraceae con 12 especies. Mientras que en el relicto 2 (predio El Topacio) se hallaron 161 especies pertenecientes a 49 familias, en donde las familias con mayor cantidad de especies fueron Fabaceae con 15 especies, Lauraceae y Burseraceae con 10 especies cada una. Por último, en el relicto 3 (predio Asoprocaucho), se encontraron 151 especies forestales distribuidas en 47 familias, Fabaceae con 16 especies, Lauraceae con 10 especies y Moraceae con 9 especies fueron las familias con mayor cantidad de especies.

Tabla 4. Métricas de paisaje a nivel de clase para el análisis multitemporal del uso del suelo en El Retorno (Colombia).

\begin{tabular}{|c|c|c|c|c|c|c|c|c|c|c|c|c|}
\hline \multirow{2}{*}{ Métrica } & \multicolumn{6}{|c|}{ Año 1990} & \multicolumn{6}{|c|}{ Año 2016} \\
\hline & AA & AAH & VS & B & OT & $\mathbf{P}$ & AA & AAH & VS & B & OT & $\mathbf{P}$ \\
\hline \multicolumn{13}{|c|}{ Métricas de área } \\
\hline Area MN (ha) & 12.0 & 10.2 & 23.2 & 918.9 & 36.2 & 24.3 & 11.8 & 9.9 & 14.8 & 94.7 & 59.6 & 128.3 \\
\hline NP (\#) & 133.0 & 52.0 & 520.0 & 62.0 & 1.0 & 552.0 & 102.0 & 51.0 & 549.0 & 339.0 & 1.0 & 332.0 \\
\hline $\mathrm{PD}(\# / 100 \mathrm{ha})$ & 0.2 & 0.1 & 0.6 & 0.1 & 0.0 & 0.7 & 0.1 & 0.1 & 0.7 & 0.4 & 0.0 & 0.4 \\
\hline LPI (\%) & 0.1 & 0.1 & 0.5 & 64.8 & 0.0 & 0.9 & 0.1 & 0.1 & 0.1 & 15.1 & 0.1 & 11.8 \\
\hline \multicolumn{13}{|c|}{ Métricas de forma } \\
\hline Para MN & 224.0 & 269.9 & 234.1 & 206.5 & 174.1 & 222.0 & 238.6 & 304.4 & 290.6 & 226.6 & 125.9 & 194.4 \\
\hline Para SD & 81.9 & 71.5 & 80.6 & 84.7 & 0.0 & 82.3 & 98.9 & 100.9 & 106.3 & 89.2 & 0.0 & 92.2 \\
\hline Para CV (\%) & 36.5 & 26.5 & 34.4 & 41.0 & 0.0 & 37.1 & 41.5 & 33.1 & 36.6 & 39.4 & 0.0 & 47.4 \\
\hline \multicolumn{13}{|c|}{ Métricas de agregación } \\
\hline ENN MN (m) & 813.4 & 921.9 & 219.0 & 124.9 & N/A & 243.3 & 963.6 & 1430.4 & 232.1 & 132.3 & N/A & 99.4 \\
\hline ENN CV (\%) & 84.0 & 93.9 & 91.0 & 66.4 & N/A & 85.6 & 98.4 & 102.1 & 90.5 & 63.0 & $N / A$ & 74.0 \\
\hline $\mathrm{Al}$ & 96.2 & 87.0 & 90.1 & 88.4 & 84.0 & 91.6 & 93.4 & 91.8 & 83.1 & 86.8 & 82.3 & 94.3 \\
\hline
\end{tabular}

Clases de cobertura: AA: áreas abiertas, sin o con poca vegetación. AAH: áreas agrícolas heterogéneas. VS: vegetación secundaria o en transición; B: bosques. OT: otras tierras. SD: sin datos.

Métricas: variabilidad de área (Area), número de parches (NP), densidad de parches (DP), índice del parche más grande (LPI), índice de forma (Shape), proporción perímetro/área (Para), distancia euclidiana al vecino más cercano (ENN), índice de agregación (Al).

* Se calcularon: media (MN), desviación estándar (SD) y el coeficiente de variación (CV). 


\section{Parámetros estructurales}

En la figura 4 se muestra la distribución por clases diamétricas de las especies arbóreas registradas, la cual es en "j invertida", concentrando la mayor cantidad de individuos en las clases diamétricas
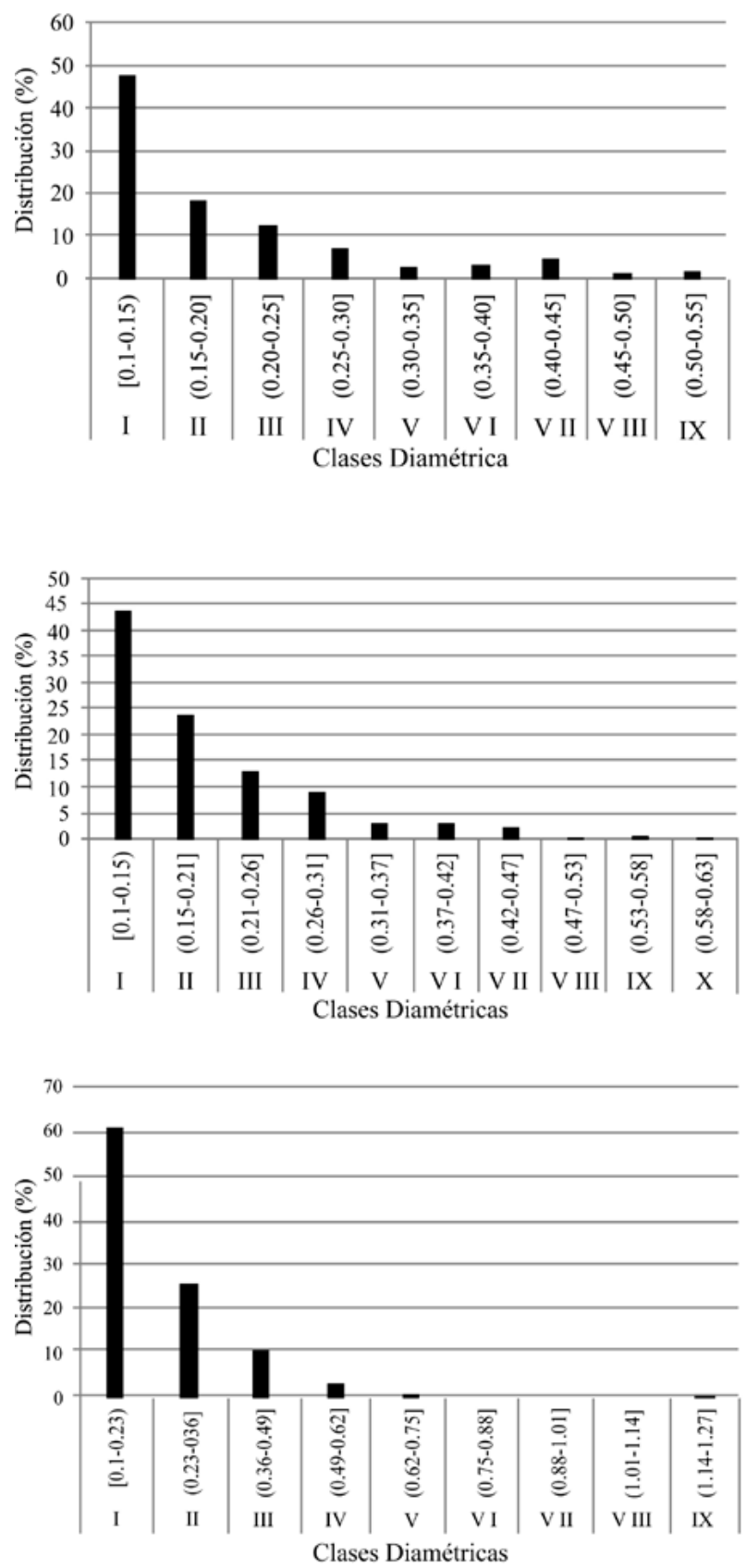

inferiores, estructura característica de bosques tropicales disetaneos. En el relicto 1, el $43.9 \%$ de los individuos se encuentran en la clase diamétrica I, con diámetros ente 10 y $15 \mathrm{~cm}$, y no se registraron árboles con diámetros superiores a los 63 $\mathrm{cm}$. El $61.01 \%$ de los individuos del relicto 2 se
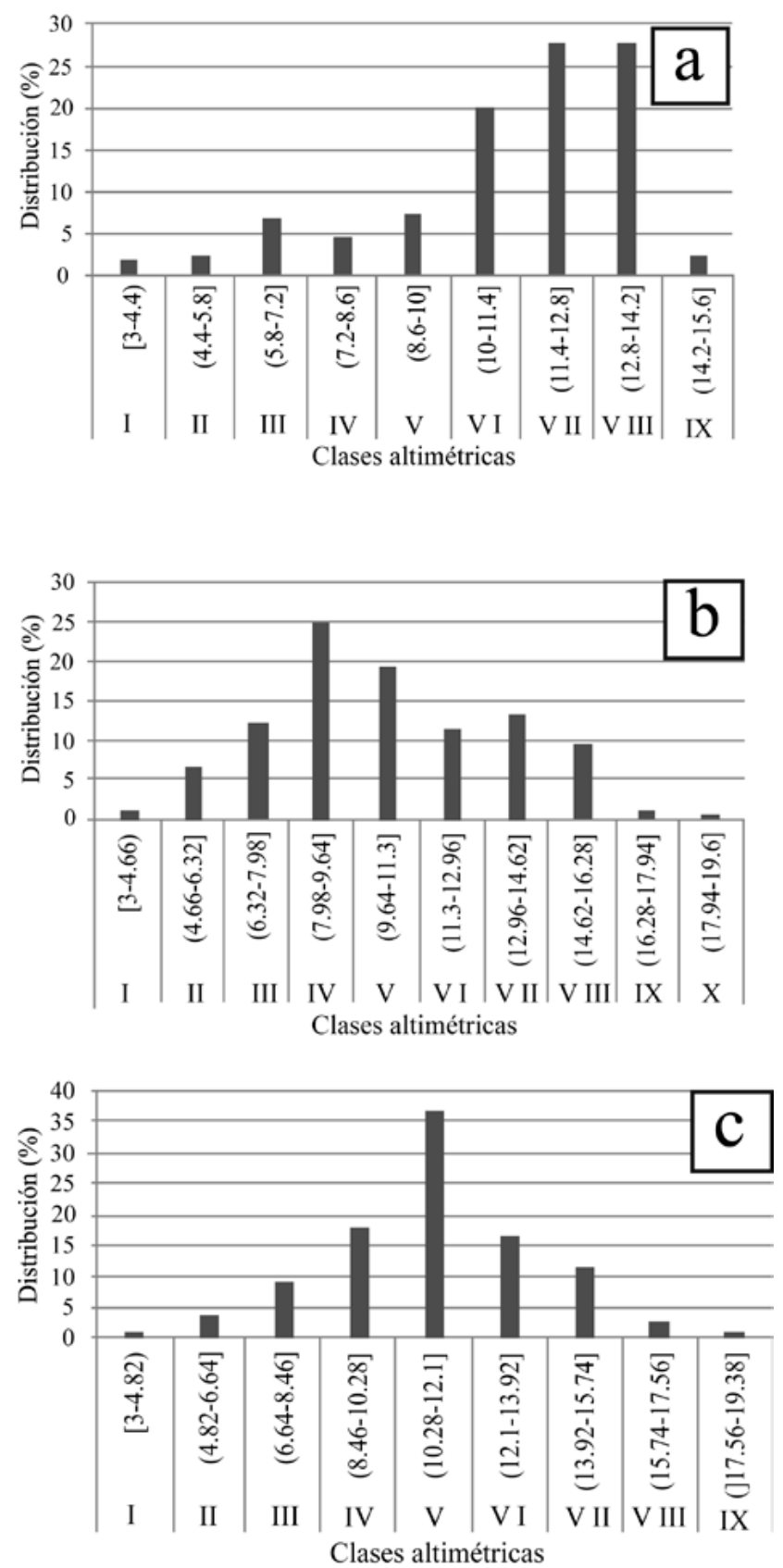

a: Relicto 1. Predio el Mirador. B: Relicto 2: PredioEl Topacio. C: Relicto 1. Predio Asoprocaucho.

Figura 4. Distribución diamétrica y altimétrica de los individuos con un DAP $\geq 10 \mathrm{~cm}$. 
encuentran en la clase diamétrica I, con un rango diamétricos entre los 10 y $23 \mathrm{~cm}$, y tan solo un $13.3 \%$ de los individuos tienen diámetros que superan los $36 \mathrm{~cm}$. Por último, en el relicto 3 no se registraron diámetros superiores a los $55 \mathrm{~cm}$ y el $48.02 \%$ de los fustales se encuentran en la clase diamétrica I, con diámetros entre los 10 y $15 \mathrm{~cm}$.

En lo referente a la distribución altimétrica, la distribución de alturas es bastante heterogénea. En el relicto 1 no se diferencia un estrato con alturas superiores a los $19 \mathrm{~m}$; el 25\% de los individuos registrados en este relicto se encuentran en la clase altimétrica IV con alturas entre los 7.98 y 9.64 m y solo el $1.5 \%$ de los individuos presentan alturas mayores a los $16.28 \mathrm{~m}$. En el relicto 2 no se diferencia un estrato con alturas superiores a los $19 \mathrm{~m}$ y el $36.70 \%$ de los individuos se encuentran en la clase altimétrica $V$, con alturas entre los 10.28 y $12.1 \mathrm{~m}$. El $55.36 \%$ de los individuos del relicto 3 se encuentran en las clases altimétricas VII y VIII con alturas entre los 11.4 m y 14.2 m; no se registraron individuos con alturas superiores a los $15.6 \mathrm{~m}$.

En relación a la estructura vertical y horizontal de los bosques, se encontró que el relicto 1 presenta un sotobosque que está dominado por especies de hábito herbáceo, siendo la más abundante la Asteraceae, Pseudelephantopus sp.1, y los pastos (Poaceae), Olyra sp.1 y Olyra latifolia L.; también se encontraron cuatro especies de helechos (Pteridaceae) del genero Adiantum, A. sp.1, Adiantum cf. decoratum Maxon \& Weath., Adiantum obliquum Willd y A. sp. 4, así como una especie de Araceae del género Philodendron. En el sotobosque del relicto 2 priman las especies herbáceas, siendo la especie más abundante y frecuente la herbácea arbustiva Solanum jamaicense Mill. También se destacan cinco especies de la familia Araceae: Monstera sp.1, Philodendron sp. 1, Philodendron sp.2, Spathiphyllum cf. cannifolium (Dryand.) Schott y Spathiphyllum sp.1; así como un helecho del género Adiantum (Pteridaceae). Por último, la especie $S$. cf. cannifolium de la familia Araceae, y dos helechos del género Adiantum
(Pteridaceae), A. obliquum y Adiantum sp.3, son las especies que registran mayor abundancia en el sotobosque del relicto 3 . En la tabla 5 se mencionan las especies más representativas de cada relicto en el índice de valor de importancia ampliado.

\section{DISCUSIÓN}

La fragmentación del bosque inicia con el establecimento de cultivos de coca, evidenciado en series de tiempo detalladas que demuestran luego la aparición de pasturas sobre las áreas boscosas (Armenteras et al., 2006). Esta conversión de bosques a pastizales es una de las principales causas de cambio sobre la cobertura vegetal en la región amazónica (Dávalos et al., 2014; Rufin, Müller, Pflugmacher y Hostert, 2015; Navarrete, Sitch, Aragão, Pedroni y Duque, ,2016), que se caracteriza por tener un $80 \%$ de la superficie de pastoreo, con fincas que implementan un sistema extensivo de ganadería con capacidad de carga aproximada de una cabeza de ganado forrajero por hectárea de pasto (Navarrete et al., 2016).

Lo anterior se refleja en el área de estudio, ya que el análisis multitemporal y las entrevistas con los actores claves de la región muestran una trayectoria de intensidad histórica que sugiere un uso intensivo del suelo con grandes extensiones de pastos dedicados a la ganadería extensiva y la presencia de cultivos ilícitos en áreas de bosque. Esta situación impulsó la reducción del área de las masas boscosas, lo que genera un conflicto de uso del suelo asociado a procesos de praderización.

Igualmente, los resultados presentes en este trabajo confirman que la expansión lateral para reducir al mínimo la distancia de las zonas productivas de los pastos forma un patrón especifico que está directamente relacionado con altos índices de fragmentación de los bosques (Peng et al., 2016). Por ende, es un determinante de grandes cambios en las medidas de paisaje al convertirlo más simple y homogéneo, confirmando así un proceso de consolidación de pastizales. Esto se evidencia en 
Tabla 5. Indice de valor de importancia ampliado para relicto de bosques en El Retorno (Colombia).

\begin{tabular}{|c|c|c|c|c|}
\hline Especie & IVI (\%) & PS (\%) & RN (\%) & Ivia $(\%)$ \\
\hline \multicolumn{5}{|c|}{ Relicto 1. Predio El Mirador } \\
\hline Siparuna cuspidata (Tul.) A.DC. & 23.01 & 1.37 & 0.82 & 25.20 \\
\hline Astrocaryum chambira Burret & 14.62 & 2.50 & 1.73 & 18.85 \\
\hline Schefflera morototoni (Aubl.) Maguire et al. & 12.94 & 0.92 & 0.00 & 13.86 \\
\hline Vismia macrophylla Kunth & 7.91 & 3.34 & 2.13 & 13.38 \\
\hline Inga acreana Harms & 10.52 & 0.99 & 0.90 & 12.41 \\
\hline Virola peruviana (A.DC.) Warb. & 4.89 & 2.71 & 2.44 & 10.04 \\
\hline Crepidospermum rhoifolium (Benth.) Triana \& Planch. & 6.04 & 1.80 & 1.69 & 9.53 \\
\hline Pourouma bicolor Mart. & 5.85 & 1.44 & 1.52 & 8.81 \\
\hline Miconia truncata Triana & 2.65 & 3.10 & 3.05 & 8.80 \\
\hline Oenocarpus bataua Mart. & 7.19 & 0.95 & 0.60 & 8.74 \\
\hline Protium amazonicum (Cuatrec.) Daly & 6.14 & 1.32 & 0.99 & 8.45 \\
\hline Iryanthera paraensis Huber & 6.41 & 1.03 & 0.66 & 8.10 \\
\hline Guatteria sp. 2 & 3.86 & 2.15 & 2.06 & 8.07 \\
\hline Otras especies & 187.96 & 76.39 & 81.40 & 345.75 \\
\hline Total & 300.00 & 100.00 & 100.00 & 500.00 \\
\hline \multicolumn{5}{|c|}{ Relicto 2. Predio El Topacio } \\
\hline Inga acreana Harms & 41.00 & 5.58 & 2.94 & 49.52 \\
\hline Triplaris americana L. & 40.39 & 1.42 & 0.00 & 41.81 \\
\hline Hevea brasiliensis (Willd. ex A. Juss.) Müll. Arg & 18.22 & 0.59 & 0.00 & 18.81 \\
\hline Guatteria cf. metensis R.E.Fr. & 6.97 & 4.84 & 4.32 & 16.12 \\
\hline Oenocarpus bataua Mart. & 10.70 & 2.19 & 1.97 & 14.86 \\
\hline Iriartea deltoidea Ruiz \& Pav. & 11.82 & 1.41 & 1.07 & 14.29 \\
\hline Himatanthus articulatus (Vahl) Woodson & 6.42 & 3.24 & 2.91 & 12.58 \\
\hline Inga cf. heterophylla Willd. & 6.45 & 3.09 & 2.76 & 12.31 \\
\hline Inga brachyrhachis Harms & 8.94 & 1.19 & 0.69 & 10.83 \\
\hline Protium heptaphyllum (Aubl.) Marchand & 6.99 & 1.82 & 1.80 & 10.61 \\
\hline Tabernaemontana sanano Ruiz \& Pav. & 7.93 & 1.23 & 0.99 & 10.15 \\
\hline Crepidospermum goudotianum (Tul.) Triana\& Planch. & 8.46 & 0.45 & 0.49 & 9.40 \\
\hline Otras especies & 125.70 & 72.94 & 80.07 & 278.71 \\
\hline Total & 300.00 & 100.00 & 100.00 & 500.00 \\
\hline \multicolumn{5}{|c|}{ Relicto 3. Predio Asoprocaucho } \\
\hline Triplaris americana L. & 33.61 & 8.08 & 5.92 & 47.62 \\
\hline Socratea exorrhiza (Mart.) H. Wendl. & 15.70 & 1.78 & 1.57 & 19.04 \\
\hline Apeiba glabra Aubl. & 9.01 & 2.51 & 2.21 & 13.72 \\
\hline Himatanthus articulatus (Vahl) Woodson & 9.79 & 1.35 & 1.12 & 12.27 \\
\hline Petrea volubilis L. & 0.00 & 6.23 & 4.66 & 10.90 \\
\hline Pseudolmedia laevis (Ruiz\& Pav.) J.F.Macbr. & 6.37 & 2.08 & 2.09 & 10.53 \\
\hline Astrocaryum chambira Burret & 7.69 & 1.50 & 1.24 & 10.43 \\
\hline Miconia elata (Sw.) DC. & 3.70 & 3.36 & 3.11 & 10.17 \\
\hline Mabea piriri Aubl. & 0.00 & 5.22 & 4.61 & 9.83 \\
\hline Brosimum guianense (Aubl.) Huber & 6.59 & 1.50 & 1.48 & 9.57 \\
\hline Inga brachyrhachis Harms & 6.57 & 0.92 & 1.00 & 8.49 \\
\hline Crepidospermum goudotianum (Tul.) Triana\& Planch. & 6.53 & 1.01 & 0.88 & 8.43 \\
\hline Schefflera morototoni (Aubl.) Maguire et al. & 6.97 & 0.48 & 0.32 & 7.77 \\
\hline Otras especies & 187.48 & 63.99 & 69.77 & 321.24 \\
\hline Total & 300.00 & 100.00 & 100.00 & 500.00 \\
\hline
\end{tabular}


la zona de estudio con el aumento en el área de parches de pastos y una disminución en la distancia euclidiana, así como el número de los mismos para reflejar un patrón más agregado. En el caso de bosques, se dio un aumento en la división de la cobertura, representada en un mayor número de parches y sumado al aumento de la distancia euclidiana al vecino más cercano, disminuyó su área y reflejan una mayor dispersión de los relictos de bosques.

Los bosques que se mantienen en el área reflejan un patrón de distribución lineal, lo cual se debe a que en las prácticas de uso del suelo de la región solo se mantienen en su mayoría los bosques asociados a los cursos de agua. Cabe resaltar la presencia de un parche nodal que ocupa el $15.06 \%$ de la ventana de estudio, y aunque se desconoce la calidad ecológica de este fragmento, este es de gran importancia no solo por su tamaño sino porque contribuye a la conectividad física al estar unido a bosques de galería. Además, la superficie comprendida por los elementos nodales es inferior al 30\% de la superficie total estudiada, por lo que la organización de los elementos del paisaje es el factor clave para la conservación de la diversidad (Morera, Pintó y Romero, 2007).

Sin embargo, las superficies boscosas del área presentaron un aumento significativo en la relación entre su área y perímetro, generando formas menos compactas que afectan la conservación de la diversidad al interior de los relictos (Groom, Gary y Carroll, 2006), dado que los fragmentos quedan expuestos a una mayor influencia de los ambientes periféricos (Gurrutxaga y Lozano, 2008). En este caso corresponden a una matriz de pastos, por lo que un factor clave para la manutención de la diversidad es la adaptación de las diferentes especies en el mosaico paisajístico (Morera et al., 2007).

Considerando el cambio en la configuración del paisaje en relación a la superficie de las áreas boscosas y sus valores en los índices de forma y agregación, así como inmersión de los bosques en una matriz de pastos limpios y fragmentados por cultivos de coca, puede deducirse que es un paisaje fragmentado y por ende se pueden presentar de forma simultanea efectos asociados. La fragmentación de los bosques tiene un impacto sinérgico sobre la estructura, composición y funcionalidad del bosque (Hernández, Delgado, Meier y Durán, 2012), lo que se evidencia en la caracterización de la composición y estructura de los relictos boscosos analizados, en los que se registró una mayor abundancia, frecuencia y dominancia de especies heliófitas generalistas con una escasez de especies especialistas y ausencia de grandes árboles emergentes. Lo anterior, coincidiendo con lo reportado por Stevenson y Rodríguez (2008) al describir otros relictos de bosque húmedo tropical en el municipio de El Retorno, en el que el 25\% de las especies registradas son pioneras y su mayor proporción puede deberse a procesos antrópicos de uso y fragmentación.

A pesar de lo anterior, se registraron altos valores de IVI en bosques con fines de protección o con menos alteraciones aledaños, con especies: B. guianense, C. goudotianum, C. rhoifolium, I. deltoidea, O. bataua, P. bicolor, P. laevis y $S$. exorrhiza (Duque, Cárdenas y Rodríguez, 2003; Stevenson, Suescún y Quiñones, 2004; García-R y Galindez, 2011). Sin embargo, en los bosques tropicales disetaneos el mayor peso ecológico (IVI) está dado por pocas especies con individuos de alturas y diámetros altos (Ariza, Toro y Lores, 2009). Caso contrario a lo que se registra en el área de estudio, en donde las especies con mayor IVI deben sus valores de dominancia a la abundancia de individuos en las clases diamétricas inferiores. Esto, sumado al empobrecimiento del estrato superior arbóreo, se refleja en mayor abundancia de individuos en los estratos inferior y medio $(\mathrm{HT}<15 \mathrm{~m})$, sugiriendo una simplificación estructural del bosque.

Dado que los cambios en la composición de la vegetación referidos a mayor abundancia de especies heliófitas con maderas de menor densidad, sumado a cambios estructurales, representados 
en la mayor densidad de árboles con troncos más delgados, disminuyen el tiempo de residencia del carbono en la madera y aceleran la descomposición, también se pueden estar viendo afectados los procesos ecológicos como, por ejemplo, el flujo de carbono del suelo (Laurance et al., 2011; Romero-Torres y Varela, 2011; Barros y Fearnside, 2016). Por lo cual la composición y estructura de los relictos analizados coincide con la de sitios alterados o fragmentos en los cuales se ha registrado mayor afección del hábitat (e.g. Laurance, Vasconcelos y Lovejoy, 2000; Lezcano, Finegan, Condit y Delgado, 2001; Hernández et al., 2012), respaldando la pérdida de resilencia ecológica en paisajes hiperfragmentados y la conversión hacia un paisaje secundario (Pardini, Bueno, Gardner, Prado y Metzger, 2010; Hernández et al., 2012).

\section{CONCLUSIONES}

Debido a que la dinámica de cambio de los ecosistemas boscosos es muy alta, se hace necesario el empleo de metodologías con escalas temporales y espaciales múltiples. Sin embargo, no son usuales los estudios que consideren ambos aspectos, pero este en particular, permite continuar avanzando en el conocimiento de la interrelación de las causas de deforestación a nivel de paisaje, las cuales comúnmente son precursoras de la fragmentación y degradación de bosques.

Es así como el análisis de algunos aspectos relacionados a la interacción de factores de intervención antrópica como lo es el uso de suelo, métricas de paisaje y estudios de vegetación en relictos de bosque permiten ampliar el escenario para lograr identificar si se están presentando procesos de recuperación o degradación en los bosques, para así poder definir estrategias adecuadas de manejo con fines de conservación.

La fragmentación de los bosques en el área de estudio es evidente, se refleja en el mayor número de parches con menor área y en el aumento de la distancia entre los mismos, mientras que se han ampliado los pastizales aumentando su conectividad; situación que afecta a las comunidades vegetales de los relictos de bosque presentes al estar inmersos en una matriz de pastos y al mostrar cambios en la composición de especies y estructura del bosque que actúan como indicadores de degradación forestal.

Aun así, los fragmentos de bosques que persisten en estos paisajes fragmentados constituyen áreas con gran potencial para la restauración ecológica, considerando que se mantienen valores significativos de diversidad de especies asociados a riqueza y que aún se registran especies representativas del bosque húmedo tropical que se encuentran catalogadas dentro de alguna categoría de amenaza, como lo son: las palmas, Astrocaryum chambira Burret, Euterpe precatoria Mart., Iriartea deltoidea Ruiz \& Pav. y Oenocarpus bataua Mart.; especies de la familia Lauracea como Ocotea aciphylla (Nees \& Mart.) Mez, Ocotea bofo Kunth, Ocotea longifolia Kunth; las Lecythidaceas Cariniana pyriformis Miers, Couroupita guianensis Aubl., Eschweilera gigantea (R.Knuth) J.F.Macbr y Gustavia poeppigiana O.Berg; y Cedrela odorata L. (Meliaceae), entre otras.

A pesar que los relictos de bosques estudiados han presentado pérdidas significativas de área, se mantienen áreas que desempeñan un rol fundamental en la conservación de la biodiversidad. Aun así, se sugiere la ampliación de las zonas de bosques para el mantenimiento de poblaciones y la reducción de las distancias entre fragmentos para aumentar la conectividad ecológica, así como una reducción de la matriz de pastos considerando que sin duda esta influye en el tipo de sucesión que se puede dar de forma natural en estos bosques.

Este trabajo aporta información clave para el diseño de estrategias orientadas al manejo de relictos al interior de finca, que se puedan vincular al diseño de herramientas de gestión y conservación a nivel de paisaje. 


\section{AGRADECIMIENTOS}

Los autores agradecen a The Digital Globe Foundation por suministrar las fotografías aéreas de alta resolución para el desarrollo de este trabajo. A la familia Rincón y a Asoprocaucho, por permitirnos desarrollar esta investigación en sus predios y abrirnos las puertas de sus hogares. A los ingenieros forestales Alejandra Reyes y José Luis Acosta, a la estudiante Katherine Lezama y Dairo Gutiérrez Rincón, por su apoyo en las actividades de campo; así como al profesor Gilberto Emilio Mahecha y los profesionales del herbario UDBC de la Universidad Distrital Francisco José de Caldas por la colaboración en la determinación del material vegetal.

\section{CONFLICTO DE INTERESES}

Los autores declaran no tener conflicto de intereses.

\section{CONTRIBUCIÓN POR AUTOR}

Los autores son los únicos responsables de la obra en todos los aspectos que condujeron a la elaboración de su publicación.

\section{REFERENCIAS BIBLIOGRÁFICAS}

Acharya, K. P., Dangi, R. y Acharya, M. (2011). Análisis de la degradación del bosque en Nepal. Unasylva, 238(62), 31-38.

Aguilera, F. y Botequilha-Leitão, A. (2012). Selección de métricas de paisaje mediante análisis de componentes principales para la descripción de los cambios de uso y cobertura del suelo del Algarve, Portugal. Revista Internacional de Ciencia y Tecnología de la Información Geográfica, GeoFocus, 12, 93-121.

Arancibia-Arce, L., Perotto-Baldivieso, H., Furlán, J., Castillo-García, M., Soria, L. y Rivero-Guzmán, K. (2013). Evaluación espacial y temporal de fragmentación y conectividad para actividades ecoturísticas en un sitio Ramsar: Los Bañados de Isoso (Santa Cruz, Bolivia). Ecología en Bolivia, 48(2), 87-103.

Ariza, W., Toro, J. y Lores, A. (2009). Análisis florístico y estructural de los bosques premontanos en el municipio de Amalfi (Antioquia, Colombia). Colombia Forestal, 12(1), 81-102. DOI: https://doi. org/10.14483/udistrital.jour.colomb.for.2009.1.a07

Armenteras, D., Rudas, G., Rodríguez, N., Sua, S. y Romero, M. (2006). Patterns and causes of deforestation in the Colombian Amazon. Ecological Indicators, 6, 353-368. DOI: https://doi.org/10.1016/j. ecolind.2005.03.014

Armenteras, D., Gonzales, T. y Retana, J. (2013). Forest fragmentation and edge influence on fire occurrence and intensity under different management types in Amazon forests. Biological Conservation, 159, 73-79. DOI: https://doi.org/10.1016/j.biocon.2012.10.026

Armenteras, D. y Rodríguez, N. (2014). Dinámicas y causas de deforestación en bosques de Latinoamérica: una revisión desde 1990. Colombia Forestal, 17(2), 233-246. DOI: https://doi.org/10.14483/ udistrital.jour.colomb.for.2014.2.a07

Armenteras, D. y Vargas, O. (2016). Patrones del paisaje y escenarios de restauración: acercando escalas. Acta Biológica Colombiana, 21(1), 229-239. DOI: https://doi.org/10.15446/abc.v21n1Supl.50848

Armenteras, D., Espeltab, J. M., Rodriguez, N. y Retana, J. (2017). Deforestation dynamics and drivers in different forest types in Latin America: Three decades of studies (1980-2010). Global Environmental Change, 46, 139-147.

Barros, H. y Fearnside, P. (2016). Soil carbon stock changes due to edge effects in central Amazon forest fragments. Forest Ecology and Management, 379, 30-36. DOI: https://doi.org/10.1016/j. gloenvcha.2017.09.002

Bustamante, R. y Grez, A. (1995). Consecuencias ecológicas de la fragmentación de bosques nativos. Ciencia y Ambiente, 11(2), 58-63.

Cámara, R. y Díaz del Olmo, F. (2013). Muestreo en transecto de formaciones vegetales de fanerófitos y caméfitos. I- Fundamentos metodológicos. Estudios Geográficos, 74, 67-88. DOI: https://doi. org/10.3989/estgeogr.201303 
Canzio, C. (2006). Análisis de microestructura en hierro fundido nodular y evaluación de superficie de fractura en probetas de impacto Charpy mediante el uso del software Image-pro plus. Lima: Facultad de Ciencias e Ingeniería, Pontificia Universidad Católica del Perú.

Chacón, M., Harvey, C. A. y Delgado, D. (2008). Diversidad arbórea y almacenamiento de carbono en un paisaje fragmentado del bosque húmedo de la zona atlántica de Costa Rica. Comunicación Técnica. Recursos Naturales y Ambiente, 51-52, 19-32.

Chuvieco, E. (2000). Fundamentos de teledetección espacial ( $3^{a}$ ed.). Madrid: Rialp.

Confederación Cauchera Colombiana (2016). Censo de plantaciones de caucho natural (Hevea brasiliensis) a año 2015. Bogotá: Confederación Cauchera Colombiana.

Cordero, J. y Boshier, D. H. (2003). Árboles de Centroamérica: un manual para extensionistas. Turrialba, Costa Rica: Oxford Forestry Institute (OFI), Centro Agronómico Tropical de Investigación y Enseñanza (CATIE).

Costafreda, S. (2009). Análisis de la estructura de la vegetación y su relación con la ocurrencia de incendios forestales (trabajo de pregrado, Ingeniería de Montes). Universidad de Lérida, Lérida, España.

Dane, F. (2013). El desafío del desarrollo sustentable en América Latina. Rio de Janeiro, Brasil: Konrad-Adenauer-Stiftung.

Dávalos, L., Holmesb, J., Rodriguez, N. y Armenteras, D. (2014). Demand for beef is unrelated to pasture expansion in northwestern Amazonia. Biological Conservation, 170, 64-73. DOI: https://doi.org/ 10.1016/j.biocon.2013.12.018

Duque, Á., Cárdenas, D. y Rodríguez, N. (2003). Dominancia florística y variabilidad estructural en bosques de tierra firme en el noroccidente de la Amazonía Colombiana. Caldasia, 25(1), 139-152.

Fernández, V. y Silva, R. (2015). Los paisajes en movimiento. El conocimiento paisajístico de Andalucía a través de la carretera. En: J. De la Riva, P. Ibarra, R. Montorio y M. Rodríguez (eds.), Análisis espacial y representación geográfica: innovación y aplicación (pp. 953-960). Zaragoza: Departamento de
Geografía y Ordenación del Territorio, Universidad de Zaragoza.

Fuentes-Ramírez, A., Pauchard, A., Marticorena, A. y Sánchez, P. (2010). Relación entre la invasión de Acacia dealbata Link (Fabaceae: Mimosoideae) y la riqueza de especies vegetales en el centro-sur de Chile. Gayana Botánica, 67(2), 188-197. DOI: https:// doi.org/10.4067/S0717-66432010000200004

Gadow, K., Real, P. y Álvarez, J. G. (eds.) (2001). Modelización del crecimiento y la evolución de bosques. Viena: IUFRO World Series vol. 12.

García, F. (1995). Coca, guerrilla y sociedad civil en el Guaviare: regulación de conflictos y otros controles. Colombia Internacional, 29, 18-29.

García-R, J. y Galindez, L. (2011). Caracterización ecológica de la microcuenca abastecedora de agua del acueducto municipal de San José del Guaviare. Ingeniería de Recursos Naturales y del Ambiente, 10, $31-43$.

Groom, J., Gary, K. y Carroll, C. (2006). Principles of conservation biology. Sunderland, FL: Sinauer Associates Inc.

Gurrutxaga, M. y Lozano, P. (2008). Evidencias sobre la eficacia de los corredores ecológicos: ¿Solucionan la problemática de fragmentación de hábitats? $O b$ servatorio Medioambiental, 1(1), 171-183.

Hernández, L., Delgado, L., Meier, W. y Durán, C. (2012). Empobrecimiento de bosques fragmentados en el norte de la Gran Sabana, Venezuela. Interciencia, 12, 891-898.

Instituto Amazónico de Investigaciones Científicas (Sinchi) (1999). Guaviare, población y territorio. Bogotá: Ministerio del Medio Ambiente.

Instituto de Hidrología, Meteorología y Estudios Ambientales (Ideam) (2010). Leyenda nacional de coberturas de la tierra. Metodología Corine Land Cover adaptada para Colombia Escala 1: 100.000. Bogotá: Ideam.

Instituto Geográfico Agustín Codazzi (Igac) (2008). Mapa de zonificación climática de Colombia. Escala 1: 100.000. Sistema de Información Geográfica para la Planificación y el Ordenamiento Territorial (S/G-OT). Recuperado de http://sigotn.igac.gov.co/ sigotn/ 
Jiang, P., Cheng, L., Li, M., Zhao, R. y Huang, Q. (2014). Analysis of landscape fragmentation processes and driving forces in wetlands in arid areas: A case study of the middle reaches of the Heihe River, China. Ecological Indicators, 46, 240-252. DOI: https://doi.org/10.1016/j.ecolind.2014.06.026

Landis, J. y Koch, G. (1977). The measurement of observer agreement for categorical data. Biometrics, 33, 159-74. DOI: https://doi.org/10.2307/2529310

Laurance, W. F., Vasconcelos, H. L. y Lovejoy, T. E. (2000). Forest loss and fragmentation in the Amazon: implications for wildlife conservation. Oryx, 34, 39-45. DOI: https://doi.org/10.1017/S003060530003088X

Laurance, W. F., Camargoa, J., Luizão, R., Laurance, S., Pimmd, S., Bruna, E., ... y Lovejoy, T. (2011). The fate of Amazonian forest fragments: A 32-year investigation. Biological Conservation, 144, 56-67. DOI: https://doi.org/10.1016/j.biocon.2010.09.021

Lezcano, H., Finegan, B., Condit, R. y Delgado, D. (2001). Variación de las características de la comunidad vegetal en relación al efecto de borde en fragmentos de bosque Las Pavas, cuenca del Canal de Panamá. Comunicación Técnica. Revista Forestal Centroamericana, 38, 33-38.

Martino, D. (2007). Deforestación en la Amazonía: principales factores de presión y perspectivas. Revista del Sur, 169, 3-22.

Matteucci, S. (2004). Los índices de configuración del mosaico como herramienta para el estudio de las relaciones patrón-proceso. En: G. Buzai (ed.), Memorias: Primer Seminario Argentino de Geografía Cuantitativa (pp. 1-29). Buenos Aires: Grupo de Ecología del Paisaje y Medio Ambiente (Gepama).

McGarigal, K. y Marks, B. J. (1995). Fragstats: Spatial pattern analysis program for quantifying landscape structure. General Technical Report PNW-GTR-351. Portland, EE. UU: Forest Service, Pacific Northwest Research Station.

McGarigal, K., Cushman, S. A. y Ene, E. (2012). Fragstats v4: Spatial pattern analysis program for categorical maps. Computer software program. Amherst, EE. UU.: University of Massachusetts. Recuperado de http://www.umass.edu/landeco/research/fragstats/fragstats.html
Morera, C., Pintó, J. y Romero, M. (2007). Paisaje, procesos de fragmentación y redes ecológicas: aproximación conceptual. En O. Chassot y C. Morera (eds.), Corredores biológicos: acercamiento conceptual y experiencia en America (pp. 11-47). San José, Costa Rica: Imprenta Nacional.

Murcia, G. U. G., Huertas, M. C., Rodríguez, J. M. y Castellanos, H. O. (2011). Monitoreo de los bosques y otras coberturas de la Amazonia colombiana, a escala 1:100.000. Cambios multitemporales en el período 2002 al 2007. Bogotá: Instituto Amazónico de Investigaciones Científicas (Sinchi).

Navarrete, D., Sitch, S., Aragão, L., Pedroni, L. y Duque, A. (2016). Conversion from forests to pastures in the Colombian Amazon leads to differences in dead wood dynamics depending on land management practices. Journal of Environmental Management, 171, 42- 51. DOI: https://doi.org/10.1016/j.jenvman.2016.01.037

Nepstad, D., Bezerra, T., Tepper, D., McCann, K., Stickler, C., McGrath, D., ... y Ruedas, A. (2013). Cómo abordar los motores agrícolas de la deforestación en Colombia: aumentar la producción terrestre y a la vez reducir la deforestación, degradación forestal, emisión de gases de efecto invernadero y pobreza rural. Bogotá: Earth Innovation Institute.

Ormazábal, Y., Ávila, C., Mena, C., Morales, Y. y Bustos, Ó. (2013). Caracterización y cuantificación de fragmentos de bosque nativo, en un sector del secano interior de la región del Maule, Chile. Ciência Florestal, 23(3), 449-460. DOI: https://doi.org/ 10.5902/1980509810556

Pardini, R., Bueno, A., Gardner, T., Prado, P. y Metzger, P. (2010). Beyond the fragmentation threshold hypothesis: regime shifts in biodiversity across fragmented landscapes. PlosONE, 5, 1-10. DOI: 10.1371/journal.pone.0013666

Peng, Y., Mi, K., Qing, F. y Xue, D. (2016). Identification of the main factors determining landscape metrics in semi-arid agro-pastoral ecotone. Journal of Arid Environments, 124, 249-256. DOI: https:// doi.org/10.1016/j.jaridenv.2015.08.009

Potter, L. (2011). Relationship between openness to trade and deforestation: Empirical evidence from the Brazilian Amazon. Eutopía, 2, 39-54. 
Quantum GIS Development Team (2016). Quantum GIS Geographic Information System. Open Source Geospatial Foundation Project. Recuperado de http://qgis.osgeo.org

Reyes, A. (1998). La erradicación de cultivos: un laberinto. Análisis Político, 24, 66-72.

Rodriguez, W. y Nunes, A. (2016). Relationship between openness to trade and deforestation: Empirical evidence from the Brazilian Amazon. Ecological Economics, 121, 85-97. DOI: https:// doi.org/10.1016/j.ecolecon.2015.11.014

Romero-Torres, M. y Varela, A. (2011). Edge Effect on the Decomposition Process of Leaf Litter in Cloud Forest. Acta Biológica Colombiana, 16(2), 155-174.

Rufin, P., Müller, H., Pflugmacher, D. y Hostert, P. (2015). Land use intensity trajectories on Amazonian pastures derived from Landsat time series. International Journal of Applied Earth Observation and Geoinformation, 41, 1-10. DOI: https://doi.org/10.1016/j.jag.2015.04.010

Santos, T. y Telleria, J. L. (2006). Pérdida y fragmentación del hábitat: efecto sobre la conservación de las especies. Ecosistemas, 15(2), 3-12.

Simonetti, D., Marelli, A. y Eva, H. D. (2015). Impact toolbox a portable open source GIS toolbox for image processing and land cover mapping. Luxemburgo: Publications Office of the European Union.

Stehman, S. V. y Czaplewski, R. L. (1998). Design and analysis for thematic map accuracyassessment: fundamental principies. Remote Sensing Environment, 64, 331-344. DOI: https://doi.org/10.1016/ S0034-4257(98)00010-8
Stevenson, P. R., Suescún, M. y Quiñones, M. J. (2004). Characterization of forest types at the CIEM, Tinigua Park, Colombia. Field Studies of Fauna and Flora, La Macarena Colombia,14, 1-20.

Stevenson, P. R. y Rodríguez, M. (2008). Determinantes de la composición florística y efecto de borde en un fragmento de bosque en el Guaviare, Amazonia colombiana. Colombia Forestal, 11(1), 5-17. DOI: 10.14483/udistrital.jour.colomb.for.2008.1.a01

Sun, B. y Zhou, Q. (2016). Expressing the spatio-temporal pattern of farmland change in arid lands using landscape metrics. Journal of Arid Environments, 124, 118-127. DOI: https://doi.org/10.1016/j. jaridenv.2015.08.007

Thompson, I. (2011). Biodiversidad, umbrales ecosistémicos, resiliencia y degradación forestal. Unasy/va, 238(62), 25-30.

Torres-Gómez, M., Delgado, L., Martín, V. y Bustamante, O. (2009). Estructura del paisaje a lo largo de gradientes urbano-rurales en la cuenca del río Aisén (Región de Aisén, Chile). Revista Chilena de Historia Natural, 82, 73-82. DOI: https://doi. org/10.4067/S0716-078X2009000100005

Vieira, M. y Castillo, D. (2010). Designing and implementing a role-playing game: A tool to explain factors, decision making and landscape transformation. Environmental Modelling \& Software, 25, 1322-1333. DOI: https://doi.org/10.1016/j.envsoft.2010.03.015

Vergara, G. y Galloso, J. (2004). Efecto de factores físico-sociales sobre la degradación del bosque nativo. Bosque, 25(1), 43-52. DOI: https://doi. org/10.4067/S0717-92002004000100004

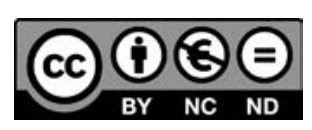

Colombia Forestal •ISSN 0120-0739 • e-ISSN 2256-201X • Bogotá-Colombia • Vol. 21 No. 2 • pp. 205-223 